\title{
Discussion on Industry Cloud Service System of SMEs
}

\author{
Xue Huang a , Liutao Zhao ${ }^{b}$ \\ Department of Beijing Computing Center, Beijing 100094, China \\ ahaungxue@bcc.ac.cn, bzhaolt@bcc.ac.cn
}

\begin{abstract}
The Industry Cloud Service is a cloud application and service platform which is built to promote the informationization of SMEs. It provides services of purchase or lease information products and integrates mechatronic products design of CAD, CAE, CAM, CAPP, PDM, and PLM with production process management. This article clarifies the importance of Industry Cloud Service from different perspectives including science and technology development, industry background and national strategy of our country. Then the concept of Industry Cloud Service is analyzed and concluded. The demand for it is elaborated from three aspects, namely function, performance and security. And the characteristics of Industry Cloud Service are summarized from construction period, function, industry model and other aspects. Premised on the basis of supporting industrial design enterprises which take design, management, and improvement of the innovation ability as the main demands, this article discusses the architecture of industrial cloud service using total-separate structure. The overall structure is mainly IaaS, SaaS and PaaS which are detailedly discussed and studied. Three different models of service procedure Industry Cloud Service provides are elaborated combining with the overall system architecture. And the Industry Cloud Service is summarized at last.
\end{abstract}

Keywords: Industry Cloud Services; Service processes; System architecture.

\section{Introduction}

During Hanover Industrial Fair on April 2013, the German government formally proposed Industry 4.0 strategy followed by a new round of industrial revolution. A production pattern of products and services with highly flexible personalization and digitization was taken as the goal of Industry 4.0. Different from the past three industrial revolutions marked by machines, electric power and information technology respectively, the fourth industrial revolution took a big step towards industrialization by taking well advantage of IoT and the expansion trend of Internet technologies into manufacturing field.

In order to achieve the strategic goal of building a manufacturing power, our state issued the "Made in China 2025" Important notice in 2015, and planned to achieve the transformation from big country to powerful country in manufacturing industry through the "three-step" strategy[1]. The formulation of "Internet + " action plan in March promoted the combination of the mobile Internet, cloud computing, big data, IoT with modern manufacturing, and facilitated the healthy development of electronic commerce and industry internet. The combinations, such as "Cloud computing + industry ", "mobile Internet + industry", "IoT + industry" and so on , provided a foundation for small and medium-sized enterprises in product innovation, intelligent manufacturing, product life cycle management, business intelligence office, promoted the deep integration of enterprise informatization and industrialization, and enhanced the core competitive advantage of enterprises.

Industry Cloud Service is a platform with cloud applications and services. It was established to promote the information technology development of small and medium-sized enterprises and to provide more convenient, secure and efficient industry cloud services. Industry Cloud Service embodies the position of the capital [3] which is "Science and Technology Innovation Center", by synthesizing innovative service capabilities and integrating advanced manufacturing capabilities.

\section{Industry Cloud Service Concept}

Industry Cloud Service could also be called industrial cloud innovative actions. Its cloud services platform is designed as a professional services platform for industrial enterprises, especially for small 
and medium-sized manufacture enterprises to provide product innovation. It covers a variety of tools and services needed by company design, manufacture, marketing, and other major product innovation processes. Based on SaaS, it provides new production services of product innovation through the Internet for customers.

Industry Cloud Service has been designed to take advantage of advanced cloud computing technologies and concepts to build a hardware platform on the basis of high-performance computer systems, data storage devices, network environment and other resources, and to encapsulate engineering calculations, industrial manufacturing, scientific computing and other mature generic application software. By the way of cloud services, it provides users with network-based, open, flexible, low-threshold and secure IaaS, PaaS and SaaS cloud services to meet the needs of various industrial enterprises, such as self-service high performance computing and parallel computing. Meanwhile it provides support in product design, testing, analysis, industrial upgrading and others for industrial enterprises. Based on the understanding of cloud service, Industry Cloud Service is a platform that takes industrial services as its major content by using cloud computing technology and related architectures.

Industry Cloud Service platform provides small and medium-sized enterprises with the purchase or lease services of information technology product and integrates mechatronic products design of CAD, CAE, CAM, CAPP, PDM, PLM with production process management [2]. It offers multi-level cloud applications information technology services utilizing high-performance computing technology, virtual reality and simulation applied technology.

\section{Needs Analysis of Industry Cloud Service}

Different from the previous industrial services model, Industry Cloud Service is a widely influential innovation service system, a new business model [3]. It meets the urgent requirement of industrial SMEs for the shared information services with high cost software and hardware resources. It can give full play to flexible features of SMEs, thus helping them to speed up the pace of innovation. Industry Cloud Service platform provides ability services, software services, consulting services, manufacturing services and other technology to reduce informatization cost, enhance collaboration of various departments, and to share social innovation resources [4].

\subsection{Functional Requirements Analysis}

Industry Cloud Service is provided with elastic computing platform, industrial design platform, enterprise management information platform, and cloud management platform. Elastic computing platform is one of the important service in Industry Cloud Service. It possesses comprehensive ability to meet all kinds of Internet users and the ability to provide users with the public Internet infrastructure service. It can effectively solve the defect of management difficulty and weak business extensibility [5] in traditional physical rent machine and VPS services. Industrial design cloud platform provides manufacturing enterprises with software and consulting services used in the product whole life cycle management including product design, modeling, simulation, data management, production process and ERP management. It is differentiated from the traditional model by expanding single service into web services. Under the mode of cloud services, business online services such as CAD online, CAE online, PDM online can be realized only by opening up the account. Enterprise management information platform meets the functional requirements of enterprises for the cooperative office software, human resources management software and customer relationship management (CRM) software which are three typical applications of enterprise management informatization. Cloud management platform achieves cloud service management of a full life cycle, including SMB management, virtual resources configuration management, user application platform building, application service release, services delivery, maintenance management and so on. 


\subsection{Performance Requirements Analysis}

Industry Cloud Service meets three categories of performance requirement: network environment requirement, virtual machine applications requirement and other requirements.

\subsubsection{High performance, high availability, high safety, manageable and extensible network environment}

High Performance - Using abundant load balancing algorithm to achieve multi-server load balancing, so that net flow can be assigned reasonable, and the overall system performance can be improved.

High Availability - The system runs stably, and single equipment failure will not affect the effective operation of the system.

High safety -- The system has excellent defense capability of DDOS attack. It provides excellent protection for the site, not only at the protocol level, but also a multi-level protection from web pages to application services and to the database. And it has good antivirus and IPS defenses function.

Manageable --The system has safe, convenient management features.

Extensible -- The system can add sites and servers as needed without interrupting other services.

\subsubsection{Reliable and stable virtual machine environments}

Infrastructure platform is upgraded and reformed so that the platform meets the reliability and stability requirements of commercial software operations. The computation and storage of cloud computing service platform, and the pooling of infrastructure resources such as network for SMEs industry can be achieved as well as enterprise-class IaaS services, such as complete control, elastic use and providing resources as needed.

\subsubsection{Other performance requirements}

Taking SMEs industrial cloud resources as support, users can visit industrial design, bioinformatics cloud platforms, and use the cloud services such as elastic computing, enterprise information management easily and fast through the Internet platform.

\subsection{Security Requirements Analysis}

Industry Cloud Service safety analysis mainly includes two aspects: data security needs and physical security requirements.

From the use of the cloud service platform of Beijing SMEs, the number of servers is very large, and the data is so dispersed. Data backup is an important way to satisfy complex data type structure and to meet the urgent need for data protection. The most import indicator of data backup is that the RTO / RPO meets the standards. RTO (Recovery Time Objective) refers to the recovery time of business operation after the disaster. RPO (Recovery Point Objective) refers to the measure standard of the acceptable amount of data in the case of the disaster. In order to give full play to the computer system performance, extend the life of the machine and to ensure data security and accuracy, it is very important to maintain good physical environmental conditions of high performance computer machine room. The entity of machine room is a complex system composed of electronic equipment, electromechanical equipment and magneto-optical material. The reliability and safety of these devices have a close relationship with environmental conditions. If the environmental conditions cannot meet the requirements of environmental equipment, it will reduce the reliability and security of computer and network devices. The damage ranges from causing data or program error, accelerating the aging of components, shortening the life of the machine to making the system break down. Even worse, it may hurt the equipment and staff.

\section{Industry Cloud Service Features}

As a cloud service support technology, Cloud computing [6] has become one of the major trends in the global IT industry. Many countries with powerful IT industry has brought it into strategic industries, and many internationally renowned IT companies also take cloud computing as important industry opportunities leading the next wave of information technology innovative. Cloud computing has brought a new revolution in information technology industry. It is generally acknowledged that 
cloud computing consists of three levels services: infrastructure as a service (IaaS), platform as a service (PaaS) and software as a service (SaaS). Industry Cloud Service Platform combines these three service patterns with industry needs and constructs an efficient flexible service platform with reasonable structure according to the actual situation of design, production and deployment of in industry. The Industry Cloud Service mainly has the following characteristics in use:

\subsection{Long Building Cycle of the Industry Cloud Service Platform}

Industry Cloud Service Platform is an innovative information service mode by combining a new generation of information technology trend with the internal demand of current industrial enterprises after deep investigation and discussion, as well as an exploratory work that can promote the integration development of manufacturing and service. Construction of Industry Cloud Service platform is a long-term task which needs to extract common things in the industry and to analyze industry structure in gathered region. So the construction, use, and promotion of the platform is a step-by-step forming ability of industry cloud service in accordance with the industry classification and the geographical promotion of application in the country or even around the world.

\subsection{Industry Cloud Service Can Promote Innovation}

Compared with traditional software, Industry Cloud Service mainly provides more value and more services for industrial enterprises by using the instantaneity of the Internet and the richness of resources. It could enhance the innovative design ability, encourage enterprises to upgrade and advance the industrial synergy developing capabilities. It could also integrate innovative design with productive resources, gather personal and business innovation fruits, encourage enterprises to obtain production tools and production data from the cloud, reduce costs and barriers of innovation and change business innovation model.

\subsection{Industry Cloud Service Creates New Model}

As an entity to show industry cloud services, Industry Cloud Service Platform provides information products hiring mode service for industrial enterprises, especially manufacturing industry, IT environmental resources services, IT infrastructure services and business consulting, solutions and other new service modes. Based on cloud technology architecture, users only need a client account and password to use the service. At the same time, it's not limited in a single process. Users can find the appropriate services in all aspects such as manufacturing, production, sales, etc. Based on MALL service model, industry cloud service has made a major breakthrough from buy to rent, from concentration to sharing, from the LAN to the Internet.

\section{The Architecture and Service Processes of Industry Cloud Service}

\subsection{Industry Cloud Service Architecture}

The ultimate goal of Industry Cloud Services of SMEs is to create an integrated, one-stop service platform by using cloud computing which is a new computing and service mode for the integrated services of industrial enterprises such as design, management, and the improvement of innovative capacity, to provide public cloud computing platform with high-performance computing, engineering computing, scientific computing and others capabilities, to provide services for large-scale concurrent users and to carry out the construction[7] according to the needs for information technology . The overall framework of Industry cloud service is shown in Figure 1. 


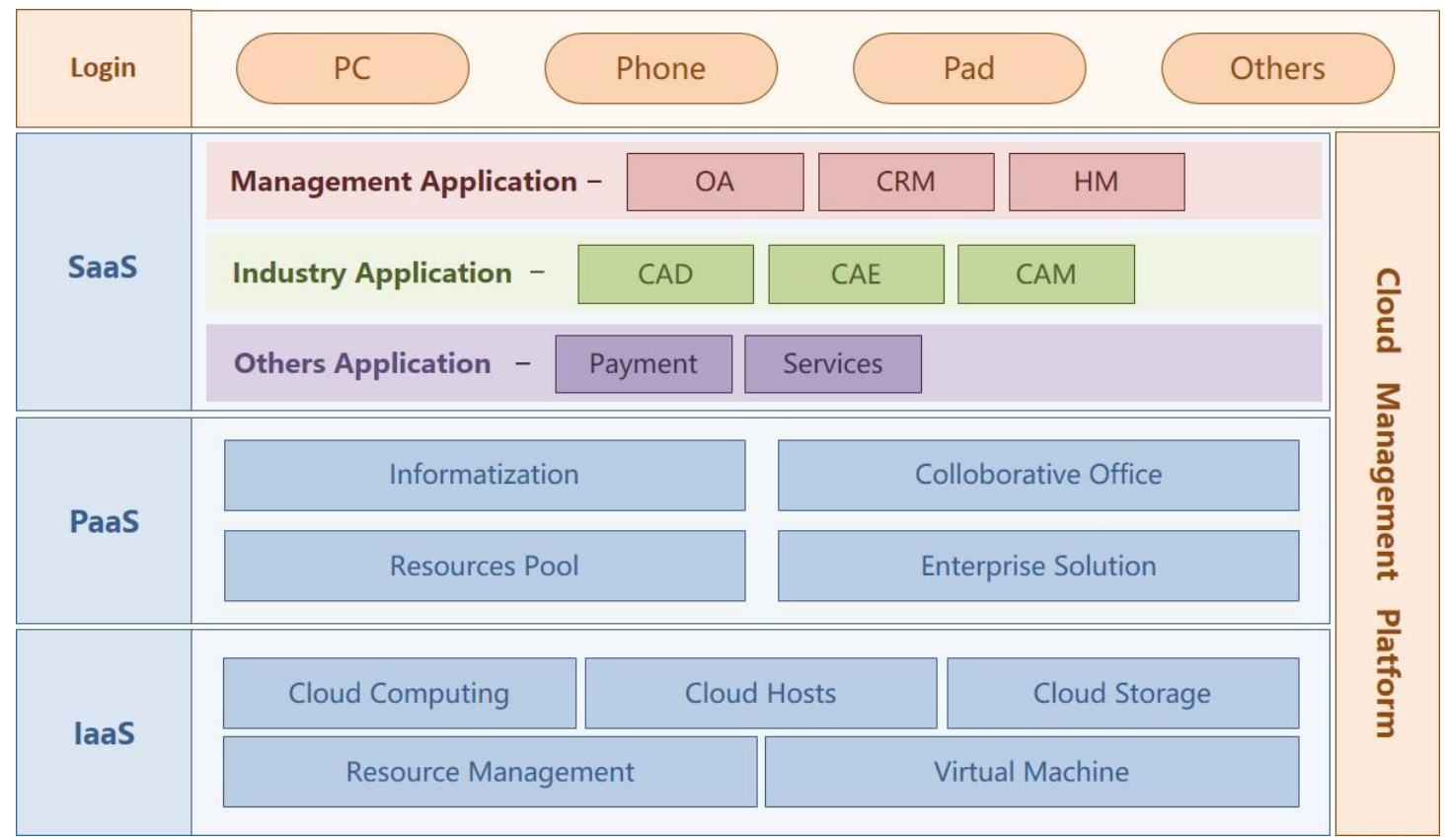

Figure 1 Industry Cloud Service Overall Framework

Combined with the actual needs of SMEs, industry cloud service architecture is shown in Figure 2. Industry Cloud Service provides three levels services for enterprises, which are basic resources rent (IaaS layer) including environmental resources and hardware resources, account lease mode service for industrial design applications (SaaS layer) and the service in which information technology is integrated into the product platform layer (PaaS layer)[8]. It is shown in Figure 2.

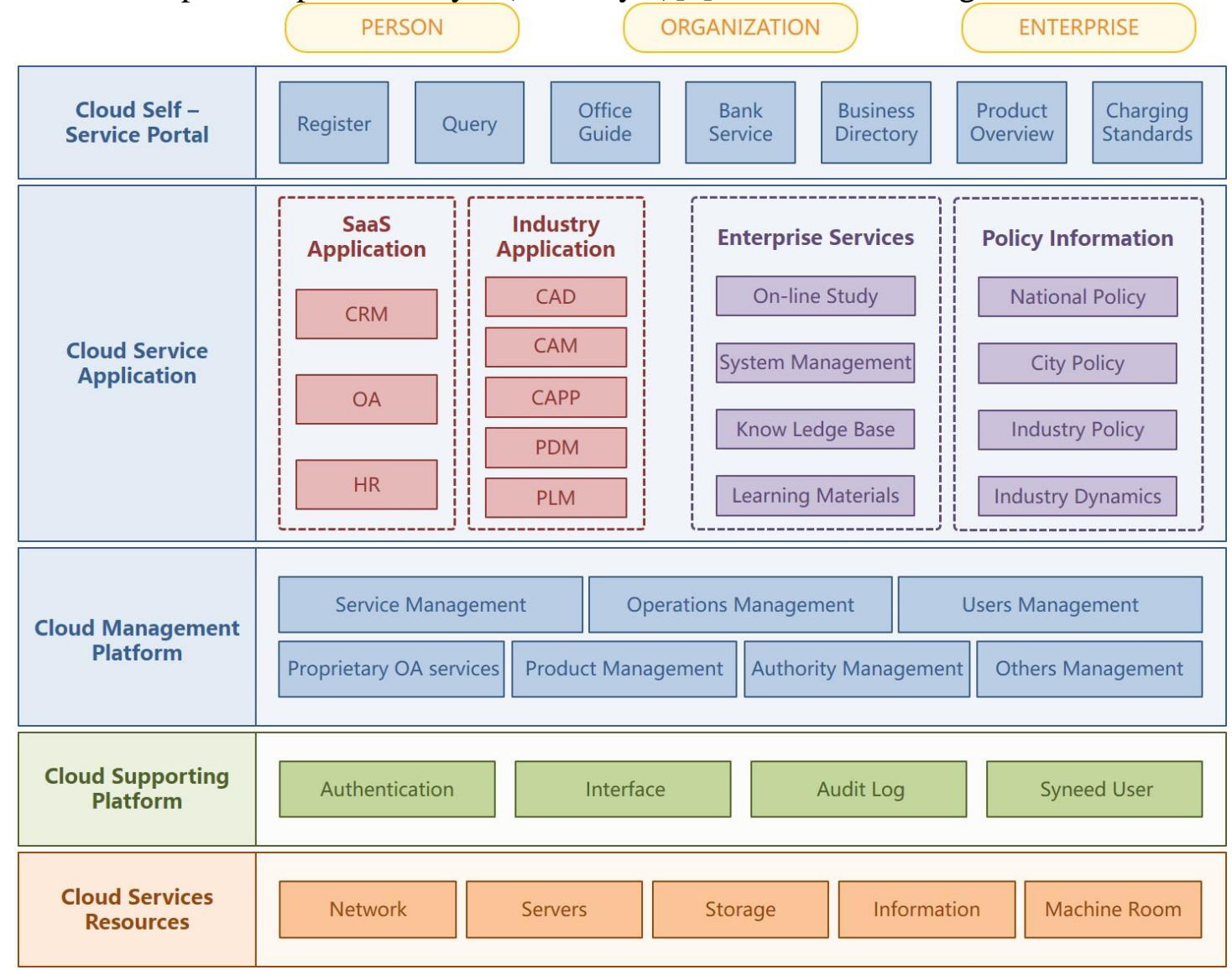

Figure 2 Industry Cloud Service Architecture

IaaS services, provided by industry cloud services (Infrastructure Service), make the infrastructure resource pooling to provide users with enterprise-class IAAS services such as flexibility in use, on-demand resources. SaaS services has integrated tools software of multispecies and multipurpose and has the ability to provide simulation, analysis, computing services for hundreds of users. 
According to users' requirements early in the design phase and refinement display, it could improve the solid structure analysis pre-processing and post-processing function of the industry design cloud platform, enhance CAE simulation analysis capabilities. From three-dimensional data processing, 3D modeling, virtual reality scenario development, display and other aspects of three-dimensional, it can enhance the service capacity of industrial design cloud platform in virtual simulation, promote design and development capabilities, and reduce cost of technological innovation. PaaS service strengthens the functions of industry cloud service for SMEs strong demand for management tools from the aspects of coordination office, human resources, In order to create a convenient and efficient use of cloud computing services for users, industrial cloud services systems also have built cloud management platform to meet the users' need that they could seamlessly connect all business system on the cloud, and deal with all business one-stop through the cloud management platform.

In order to increase the services carrying ability, availability, reliability and security, Industry Cloud Service Platform hardware supporting environment requires constant improvement by upgrading the network, purchasing servers, enlarging storage devices and storage networks, backing up data, strengthening high-performance computer room environment monitoring.

\subsection{Industrial Cloud Service Processes}

Industry 4.0 times, dramatic changes have taken place in industrial entity industry represented by manufacturing industry. Product market life cycles are getting shorter with faster renovation and more individual needs of users. In this case, it is priority to accelerate the informatization, to take advantage of internet technology to lower cost of intelligent manufacturing, smart product and on-line services and to enhance core competitiveness of enterprises.Based on technology foundation of cloud products, the platform the Industry Cloud Service has built for users is concise with clear process and transparent expenses. On the principle of on demand use, flexible computing, dynamic expansion, users could buy resources they require through the platform pages. The purchase processes of infrastructure service, cloud application service and platform-level service are similar to other business website mode. The difference is that users get virtual resources services rather than real object after days. When users of IaaS services use products of cloud storage, cloud hosts, they can configure and use resources through product web page. System will monitor the resource usage at regular intervals and draw table to feedback to the users, allowing users to understand resource utilization rates and expenses detail. Users of SaaS services purchase product authorization age and corresponding application scale in accordance with the account size required. Generally, online application uses total-sub mode, and users have an administrator account and a number of roles accounts. Administrator unifiedly deploys application resources according to the geographical position, post, deployment process. Accounts information is saved within the cloud storage device, and users can download to a local server at any time. In order to ensure data security, Industry Cloud Service Platform is also provided with disaster recovery services. PaaS service [9] is different from using a single application. Industry Cloud Service perfectly combines business application with applications platform to create resource pooling of business platform capability. What the users purchase are the business solutions of platform layer, and with no need to buy additional resources, it could complete a series of information management including internal coordination office, resource management, human resource management, customer relationship management, product management, data management and . It greatly reduces management costs, increases productivity, and improves work output.

\section{Conclusion}

As a newly developed service mode, Industry Cloud Service has been widely used in entity manufacturing industry of industrial field based on the strong support and promotion of the country and the market recognition. The following job for Industry Cloud Service is to deepen industry service content constantly and to integrate more industry needs into service platform so that it can provide 
services for more users, speed up the adjustment of economic structure of the industry in our country and transform the economic development pattern.

Industry Cloud Service can help SMEs solve multi-aspect problems in the research development innovatively such as high cost of informatization, low research efficiency, longer product design cycle and so on. It can shrink informatization "digital divide" for SMEs by providing consulting services, common technical, support-guarantee, technical exchange and efficient service. It could also accelerate the transformation and upgrading, and promote the "Made in China" to "Created in China". It has important practical significance [10].

\section{References}

[1] E-works Digital Enterprise Website. Intelligent Manufacturing:Deeper Integration of Information Technology and Industrialization Megatrends. http://articles.e-works.net.cn/view/article116283.htm

[2] 360 encyclopedia. Industry Cloud Service $\quad$ Platform. http://baike.so.com/doc/7344949-7610351.html

[3] Industry Cloud Service Platform: Promote Innovation Industry, Enhance Core Competitiveness. China Chief Information Officer Website. http://www.cio360.net/show-323-96605-1.html

[4] Yanjun Gao. Discussion on the Construction of Intelligent Public Transport Cloud Platform [J]. Electronic World.2012.6.

[5] David S. Linthicum. Write. Cloud Computing and SOA [M]. Guoyao Ma .Translate. Beijing. Posts and Telecom Press, 2011.1.

[6] Moyer, C. M. Write. Build Cloud Application: concept mode and Practice [M]. Yi Gu. etc Translate. Beijing. China Machine Press, 2012.3.

[7] Wei Qi, Bing Liu, Shihua Lu. Cloud Computing: From Infrastructure to Best Practice [M].Beijing. Tsinghua University Press, 2014.

[8] Hongmei Zhang, Changyong Liang, Jian Xu. Wisdom Tourism Cloud Services Concept Model and Service System Study [D]. Journal of Beijing University of Nationalities.2016(1).

[9] Jian Zhou, Mingxin Zhang. Cloud computing PaaS Service Architecture Research and Design [J]. Journal of Changshu Institute of Technology, 2012(26).

[10] Jietang Tian. Economic Effect and Strategic Countermeasures of China Cloud Computing [M]. Beijing. China Develop Press, 2013.6. 